\title{
Physical Emotion Induction and Its Use in Entertainment: Lessons Learned
}

\author{
Ralph Kok+, Joost Broekens* \\ *Telematica Instituut \\ Enschede, The Netherlands \\ +Media Technology / *LIACS \\ Leiden University, Leiden, The Netherlands
}

\begin{abstract}
It is well known that our emotional response is related to our bodily state, and more specifically that our bodily state can directly influence particular emotions we feel. It appears, however, that this fact has so far not had a significant influence in the entertainment industry. We first review existing work on physical emotion induction. Based on this work we present several techniques to influence emotional responses through physical means in a non-cognitive manner. The basis for the different techniques is a two-factor model of emotion: Pleasure and Arousal. We selected 4 sets of movie clips that correspond to the 4 possible quadrants existing in the 2 factor model. We have implemented some of the emotion induction techniques in a physical device (interactive chair) and tested the effects on the immersiveness of the movie clips and the emotional experience of the participants.
\end{abstract}

\section{Introduction}

Humans can be emotionally influenced in many ways. The entertainment industry makes good use of this fact by using video and audio to do so. We see a film and are grabbed by the story, we hear happy, sad or tense music and our feelings respond accordingly. There is a multitude of audiovisual means to influence our emotions, but somehow, emotion induction through physical means has been largely ignored, even though psychological research clearly shows that our emotional state is at least partially dependent on our physical state. Examples of devices that do use physical means, such as vibrating game controllers and moving seats in amusement park theatres, mostly mimic what is seen on the screen to enhance the experience and make it more immersive. They work well in achieving their goal, but they are not aimed at inducing a particular emotion, as music or storylines are. 
Here we report on research that aims to influence a person's emotions through physical means, in order to enhance the emotions experienced while watching a movie or playing a video game. To enhance rather than disrupt such an immersive experience, this process of physical emotion elicitation should happen implicitly, i.e., without the user being cognitively aware of any influence. Furthermore, we try to minimize invasiveness of the mechanisms used to achieve emotion elicitation.

We first review the concept of emotion, its relation with the bodily state, and physical emotion induction. Based on this review we present different techniques to influence particular emotions, and discuss an experiment with a physical device that implements some of these techniques. Emotional influence through audio and video is ignored in this research as it does not involve direct physical influence.

\section{The Nature of Emotion}

Emotion has long been the terrain of much research and discussion. Ever since James [1] presented a theory stating that emotions are only the result of changes in the body, the roles played by physiology and cognition in relation to emotion have been heavily debated. The current views on these relationships are described by Philippot et al. [2] in three models, the most interesting of which, for this research, is the central network model. This model argues that different emotions are associated with different changes in body state, and that changes in body state that are typical for a specific emotion will elicit that emotion and that this phenomenon, called peripheral feedback, occurs at an implicit level (i.e., without the person experiencing it being explicitly aware of the process). We focus on this model, as it is the most relevant in the context of implicit physical emotion induction.

\section{Influencing Emotion}

Facial expression It has been shown that facial expression can increase feelings of emotion [3, 4]. What is more, emotion-inducing facial expressions have been shown to be specific for specific emotions. However, physically forcing someone to have a certain facial expression in order to enhance emotional sensation is, arguably, not a good way to enhance entertainment in an unobtrusive way.

Posture The findings for emotion induction through facial expressions were hypothesized to be true for postures as well. The results of a second experiment in Duclos et al.'s research suggest that this is indeed the case, This study revealed that in some cases specific postures can be associated to fear, anger and sadness. 
In earlier research, Riskind \& Gotay [5] showed developed helplessness and stress reactions in response to posture manipulations. They showed that stress reactions can in fact occur simultaneously with a slumped-over posture, as long as an external factor explaining the emotional response is present. In the context of physical emotion induction in entertainment, this point is of great importance, because while for example one is watching a movie, an external factor would indeed be present to explain any experienced emotional response and physical posture manipulation may be used to enhance the emotional experience triggered by the audiovisual media as long as the manipulation occurs without the user being aware of it.

Respiration While Boiten et al. [6] had already found objective respiratory patterns that could be associated with specific emotions and Philippot et al. [2] showed that people can produce quite specific emotions through self-regulation of breathing patterns. These patterns were subjective, but specific, showed a clear differentiation among emotions and were consistent with the objective patterns that were found by Boiten et al.. The subjective breathing patterns found in the first study were used in a second study to induce emotions in subjects who were unaware of the goal of the study and simply followed breathing instructions.

Temperature It has been shown that temperatures of parts of the body vary in association with emotions $[7,8,9]$. McFarland has shown a relation between skin temperature changes and affect in music. The causal relationship in this case is inferred in the direction of emotion towards physical response. A reversed causal effect where skin temperature changes can cause changes in affect would be of great interest for the purposes of implicit emotion elicitation.

Such a relation has been investigated, but most investigations on this subject focus on the relation between temperature and aggression (for a review, see Anderson [10]). The basic hypothesis concerning this relation is that uncomfortably hot temperatures increase aggressive tendencies. Although findings are not fully consistent across various studies, especially not for those performed in a laboratory setting, results do clearly support the temperature-aggression hypothesis. An increase in general negative affect as a result of hot as well as cold temperatures has more recently been shown by Anderson et al. [11], but this increase was considerably less pronounced than the effect of temperature on hostility.

Haptic and Tactile Feedback In the context of video games, haptic feedback is implemented through vibrating controllers, often mimicking sound effects or other events seen on screen. It can also be used to communicate information, for example in wearable tactile displays [12]. Some interesting experiments have been performed that use haptic feedback in the communication of emotion between humans, but they are concerned with active cognitive interpretation of a communicated emotional state $[13,14,15]$. None of these implementations are used to elicit an emotional state in a user though. 
More interestingly, a product called the Hapticat was developed to study the relationship between touch and affect [16]. The product can be described as an abstract pet, communicating emotional states through various behaviors. While people were able to discern distinct emotions and the interaction changed their personal affect, the results are ambiguous when considering this for use in implicit emotion induction. Users were presented with a variety of emotions to ascribe to the 'pet', making them cognitively aware to the presence of an emotional charge. Also, the experiment did not seem to be aimed at reproducing the same emotions in the users through for example empathy, but rather influenced users' affect in terms of elation and surprise when the pet responded.

Heart rate Tactile feedback can be used to in a more specifically emotion inductive manner, namely to influence heart rate. Research indicates that false feedback of heart rate has the potential to alter our emotional response $[17,18,19]$, even when the feedback signal is ignored [20]. Although most studies do not show that false feedback of heart rate influences actual heart rate, they do show that emotional interpretation itself can change as a result of false feedback. Whether it works through a process of attribution or through a more direct and purely physical process is beyond the scope of this research to answer. What is important here is the existence of an effect.

Palmar sweat Let's now look at the phenomenon of the sweaty palm. It has been shown that skin conductance changes in response to emotion [21, 22, 23, 24, $25,26]$. Anxiety or emotional arousal affects the resistance and conductance of the skin: the higher the level of arousal, the higher the electrical conductance and the lower the resistance of the skin. The activity of the sweat glands has been associated with this effect, and is generally accepted as an index for the level of arousal $[27,28]$. Of course in this research, it is not measurement of anxiety that is of importance, but rather the induction of it. And even though no research to date has investigated emotional responses as a result rather than a cause of palmar sweat, such an implementation deserves attention. A practical advantage here is that Allen et al. [29] have shown that emotional sweating is not restricted to palms, but occurs on other parts of the body as well.

Scent Odours can have a strong influence on emotion and mood. In fact, this influence can be stronger than that of any other sense, due to a more direct linkage of the olfactory sense with the part in our brain that is "critical for emotional memory" $[30,31]$. The result is that odours can get certain meanings and evoke emotional associations, influencing our mood, emotion and performance. Epple \& Herz have shown this to be true for children who had learned to associate a certain smell with failure and Rétiveau et al. [32] showed an effect of fine fragrances on the mood of women. The keenness of our sense of smell has been further demonstrated by Haviland-Jones and McGuire [33], who showed that humans can in fact discern the emotions of fear and happiness from body odour. However, due to pratical issues we leave scent out of the equation in this research. 


\section{Components of Emotion}

How can we induce emotion through physical means and on an implicit level and what representation of emotion should be used?

One approach to answer this question would be to list the specific emotions that have been found to allow for physical elicitation. Such a list would presently consist of fear, anger, sadness and joy. A problem would occur when we try to determine which physical measures can induce which emotions, because not all types of influence mentioned yield distinct results in terms of which emotions they address.

To avoid having to list emotions together with feedback effect, we opted for a different approach. We couple various physical feedback measures to the pleasure-activation model of emotion, as used by Russell [34]. In this model, emotions consist of two dimensions: pleasure and arousal. Any emotion has at least these two components. Based on this model, we have developed ways to use physical measures to influence the emotional state on the pleasure and arousal axis. This approach has the added advantage that we need not achieve the level of specificity used in some researches to achieve one specific emotion. The goal is to stimulate certain emotional responses to enrich an experience in which emotions are already triggered, such as watching a movie. In this setting, manipulation along the two proposed axes should be sufficient to enhance the experienced emotions.

In terms of posture, both fear and anger are very tense, while sadness is related to a very relaxed posture. From this it would seem that posture is most effective in targeting the arousal component of emotion, with high arousal requiring a tense, and low arousal requiring a relaxed posture.

The indicated respiratory patterns for the emotions of joy, anger and sadness are more specific than those for postures even. The aspects that vary among the patterns are speed, amplitude and tenseness of the ribcage and shoulders. The latter is of course more of a posture manipulation. Looking purely at breathing, speed and amplitude remain as important factors, but even though by varying speed and amplitude we can influence emotion, it is unclear in which dimension. It seems that faster, shallower breaths produce high arousal and low pleasure, affecting both dimensions and that slower and deeper breathing reduces arousal, but does not necessarily increase pleasure.

Moving on to temperature, we have seen that aggression can be caused by uncomfortably hot temperatures and negative affect can be induced by hot and cold temperatures alike. Also, the other way around, skin temperature decreases as a result of music with negative affect and increases with positive music. We can conclude, then, that temperature influences the pleasure dimension of emotion when it reaches uncomfortable values, but this may also indicate that comfortable 
temperatures prevent the occurrence of negative affect and as such, unlike extreme temperatures, may be used in implicit emotion elicitation.

For false haptic heart rate feedback, things are somewhat clearer. By influencing heart rate, we influence arousal.

Then there is palmar sweat, or rather emotional sweat in general. As said, the extent to which the palms of one's hands sweat is an indication for that person's anxiety, which relates to high arousal. Therefore, if sweating can induce an emotional response, it will affect it so that arousal increases.

\section{Practical Implementation}

Now that we have an overview of changes in body state that influence emotion, we must look at how they can be implemented. Because we are looking at ways to influence emotion during a multimodal experience, such as watching a movie or playing a video game, modifying a chair to allow for manipulation of a person's body state seems like a logical choice. We now discuss how such a chair could be adapted to implement different emotion induction techniques.

First, it is wise to use a chair with an adjustable angle for the back. This can then be fitted with servomotors for example, making the angle of the back and thus the posture of the person in the chair automatically adjustable.

As easy as it seems to manipulate a person's posture, so hard is it to manipulate a person's breathing pattern. So far, in studies done on the influence of respiratory patterns on emotion, the subjects were asked to actively adjust these patterns. This is something we can not do in the context of what we're trying to achieve, so another way to influence respiration without the person being cognitively aware of it must be found. For now, we have no solutions that do not rely heavily on intuition. In the current experiment, influence of respiration will therefore not be included.

Influence of temperature seems something that should be feasible through an automated system. In a chair, we can think of heating elements and a cooling system integrated into the back and seat of the chair. Another, perhaps more convenient, way may be through the ventilation of air with varying temperatures onto one's neck.

False heartbeat feedback through haptics is something that may be achieved through attaching a set of vibrating units to the legs of the chair; when the frame of a chair vibrates, this will be felt through the entire chair. By measuring the actual heart rate of the person in the chair and adjusting the feedback to be slightly faster or slower, it should be possible to influence the actual heart rate.

As far as sweat goes, inducing or simulating palmar sweat would be ideal, but considering the fact that each person places his or her hands in different locations and positions, inducing sweatiness on a more 'stable' location of the 
body is much more convenient. In this case the back is a good candidate, as it is at all times resting against the back of the chair and apart from the palms the back is a place where we readily notice sweatiness in response to anxiety. To recreate the experience of a sweaty back, the back of the chair can be fitted with a type of irrigation system which can be triggered to release small droplets of liquid.

Summarized, this gives us the following list of modifications that could be made to the chair:

1. Motors to automatically adjust the back of the chair

2. Ventilation system venting warm or cold air onto the neck of the user and/or heating and cooling elements in the back

3. System for measuring actual heart rate of the user

4. Vibration elements attached to the chair to create heart rate feedback

5. Irrigation system in the back of the chair to moisten the back of the user

\section{Research question}

The main question that this research will attempt to answer using this prototype is if physical manipulations can increase the immersiveness of a multimodal experience.

There is much evidence to suggest that physical influence of emotion is possible, supporting the central network model of emotion, and the assumption that more intense emotional reactions to a multimodal experience increase its immersive potential leads to the expectation that the answer to this question will be positive. This leads us to the following hypothesis:

An emotionally charged multimodal experience will be subjectively experienced as being more intense when the physical states related to those emotions are induced than when this is not the case.

To test this hypothesis, we have developed a prototype interactive chair, and tested its effect on the experience subjects have while watching movies clips of different emotional content. 


\section{Materials and Methods}

Overview To reduce complexity and duration of development, the prototype chair we developed for this research uses four of the five modes of physical manipulation: posture $(1)$, heart rate $(3,4)$ and temperature $(2)$. See Fig. 1 for a view of the chair. On the left the air flow regulation system can be clearly seen, which consists of tubing connected to a fan at the lower end and opening onto the sides of the participant's neck at the front of the chair. This is used to achieve cooling of the participant to a lower, uncomfortable temperature. Heating is achieved through pads integrated in a flat cushion on the back of the chair. Also on the left picture the vibrating motor can be seen which provides heart rate feedback. It is attached to the back so that vibrations can be felt throughout the entire chair. Automated adjustment of the back for posture manipulation was implemented through an integrated motor. An Arduino I/O board connected to a computer system allowed controlling the hardware of the chair prototype.

Emotional video footage consisting of eight different scenes was shown to a total of 20 participants. The scenes were selected to fall into one of the four quadrants in the pleasure-arousal domain, each quadrant being represented by two scenes. Every participant viewed four scenes without being influenced by the chair and four while the chair attempted active influence, so that for each pleasurearousal quadrant every person would see one scene with influence and one without. The order of the scenes, as well as which were shown with and without active influence, was randomized for each participant. Each scene was shown to an equal number of participants with and without active influence. To account for a possible need of participants to adjust to the chair being active, the scenes were grouped by influence, meaning that all scenes with influence were shown consecutively, as were those without. Half of the participants started with the scenes with influence, the other half started with those without.

Subjects The subjects were 10 males and 10 females, aged between 15 and 58 years (mean age 27), 13 of which were students at the Media Technology master programme of Leiden University.

Procedure The participants, one per session, were led to the experimental setup. They were seated in the chair created for this experiment and faced a computer monitor. The experimenter explained the procedure, took place behind a folding screen and when the participant was ready, the first scene was shown on the computer monitor. After viewing the scene, the participant was presented with a questionnaire, based on the Affective Level Questionnaire [9]. The questions concerning emotions that could be clearly separated into a pleasure and an arousal component were used and intermingled with non-emotional items to obscure the actual goal of the experiment. Two items reflecting the measure of immersion of the participant into the scene were also added. Each question consisted of two 
terms located at either side of a scale of 1 to $6,3.5$ being the neutral position. A slider could be positioned at 0.1 intervals along the scale to indicate the intensity of the experienced feeling. The questions were slow heartbeat - fast heartbeat, security - anxiety, cold - hot, sadness - joy, contentedness - frustration, distraction - immersion, tired - energetic, boredom - fascination, calmness - tension and unpleasantness - pleasantness. Emotional items were picked to be at opposite sides of the pleasure-arousal domain. One extra question was added: "Try to describe in your own words what emotion(s) you experienced during this scene".

After the participant had answered the questions, the process was repeated until all eight scenes were shown and questionnaires answered. After this, some more questions followed concerning experience with movies, favourite genres, etc. Then the participants were debriefed about the goal of the experiment and had a chance to ask questions and give comments.

\section{Results}

Through the questionnaire, each participant rated the intensity of various experienced emotions for each scene. The intensity score reflected which of each set of items was relevant (smaller or larger than 3.5) and how intense it occurred. These values were corrected to reflect the direction and intensity with a number between -1.0 and 1.0 (subtract 3.5, then divide by 3.5 ).

To analyze the intensity of emotions experienced with and without influence of the chair, the scores for the items reflecting axes in the pleasure-arousal domain were used. The calmness-tension item reflected purely the arousal axis,
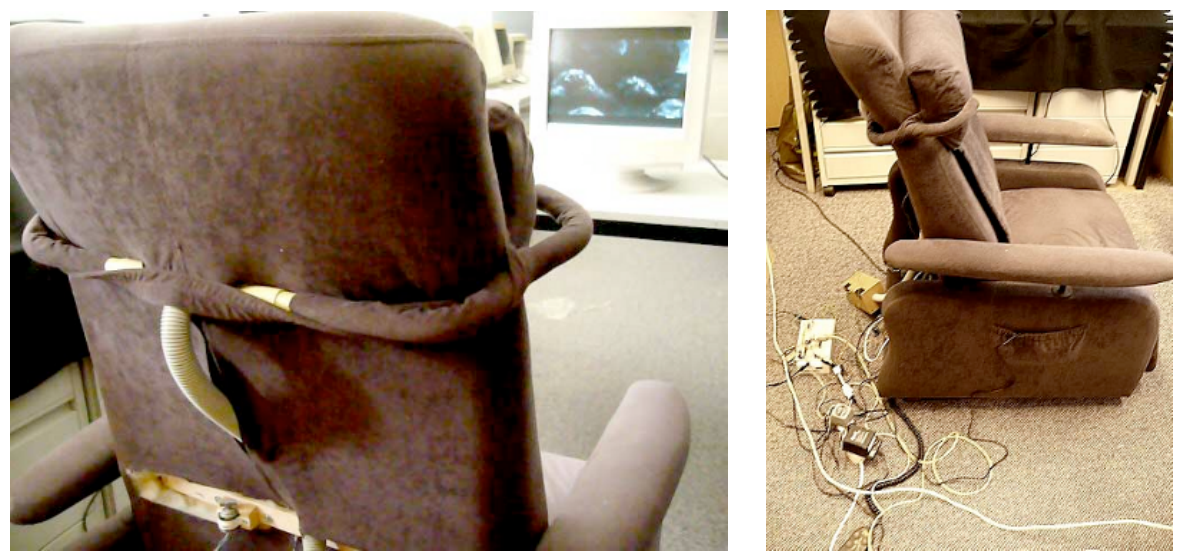

Fig. 1. Two views of the chair prototype that was developed for this experiment. On the left the air ventilation system as well as a vibration feedback implementation can be seen. The picture on the right shows the back cushion into which heating pads are integrated and the electronics that communicate with the hardware. 
the unpleasantness-pleasantness item the pleasure axis, and sadness-joy and contentedness-frustration two pleasure-arousal diagonals. Pleasure and arousal intensity values were extracted from the scored intensities for these items and were used to map the average locations in the pleasure-arousal domain at which the different scenes were rated, with and without chair influence.

Fig. 2 shows how the various scenes were rated in terms of pleasure and arousal, with a distinction between scenes seen with and without chair influence. Scenes 1 and 2 were chosen to represent quadrant 1 (top right), scenes 3 and 4 quadrant 2 (top left), scenes 5 and 6 quadrant 3 (bottom left) and scenes 7 and 8 quadrant 4 (bottom right). The graph shows the difference in reported pleasure and arousal intensity between scene presentation with and without chair influence, as well as between the different scenes.

A Bonferroni adjusted pairwise comparison of the difference in effect of the four pleasure-arousal quadrants on pleasure and arousal intensity showed that the effect on reported intensity of pleasure is not significant between pleasurearousal quadrants 1 and 4 and quadrants 2 and 3, which is to be expected as quadrants 1 and 4 are on the same side of the pleasure axis, as are quadrants 2 and 3 . Concerning the effect of the quadrants on arousal intensity a similar effect was seen, but between quadrant 1 and quadrant 3 . The expected similarity between quadrants 1 and 2 (positive arousal) and quadrants 3 and 4 (negative arousal) does not show.

Analyses of the reported emotion intensities have not shown any significant effect of chair influence on experienced intensity when split either by quadrant or scene or in aggregated tests. Some significant values were found when the separate questionnaire items were considered and analyzed per scene. Table 5 gives an overview of the $p$ values that showed significance.

\section{Average FX vs no FX}

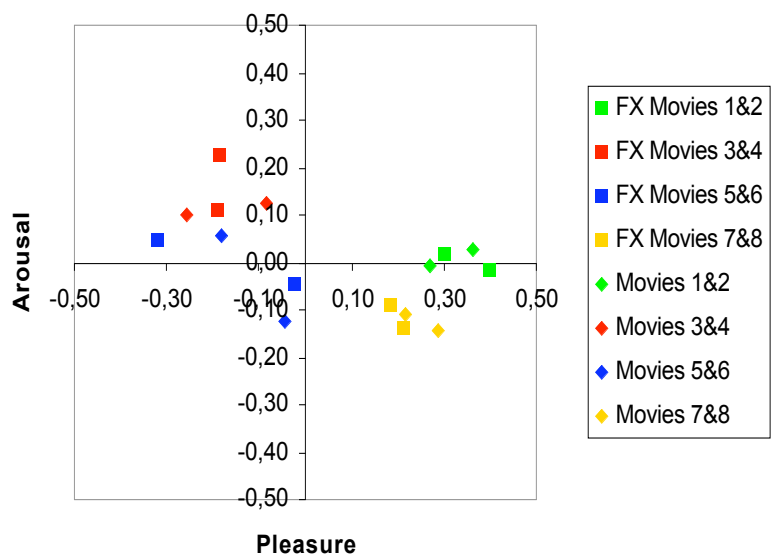

Fig. 2. Average pleasure-arousal mapping of 8 scenes, with and without chair influence (FX). Similarly colored items represent scenes chosen to fit the same pleasure-arousal quadrant. 
Table 5. Overview of $p$ values $<0,05$ for the effect of chair influence on the reported intensity for ten questionnaire items, split per scene

\begin{tabular}{lll}
\hline Question & Scene & $\mathrm{p}$ value \\
\hline Slow-fast heartbeat & 4 & 0,029 \\
Contentedness-frustration & 6 & $0,038^{*}$ \\
Distraction-immersion & 5 & 0,038 \\
Boredom-fascination & 1 & $0,034^{*}$ \\
& 2 & 0,03 \\
Unpleasantness-pleasantness & 6 & $0,038^{*}$ \\
\hline
\end{tabular}

* Mann-Whitney 2-tailed test did not show significance

\section{Discussion}

Considering the data presented in Table 5 we can conclude that this experiment did not succeed in confirming the hypothesis that an emotionally charged multimodal experience is subjectively experienced as being more intense when the physical states related to those emotions are induced than when this is not the case. Although in some cases intensities significantly differ with influence, there does not seem to be a clear effect. Only scene 1 and 2, which represent the same pleasure-arousal quadrant, show a significant effect on fascination. One might conclude from this that bodily influence did have some effect on scenes high in both pleasure and arousal, but when we look at Fig. 3 we can see that the effect on pleasure is opposite in both scenes, eliminating the possibility to draw such a conclusion.

Fig. 3 and 4 also show the division into pleasure-arousal quadrants of the scenes based on participants' ratings. It seems that in general scenes were rated as being in the quadrant they were selected to represent, with some ambiguity in scenes 5 and 6 , which corresponds with the scattering of the items for quadrant 3 seen in Fig. 2.

The design of the experiment described here has led to some choices that

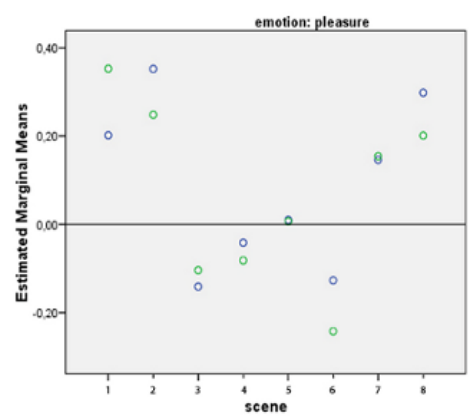

Fig. 3. Estimated means of reported pleasure intensity per scene

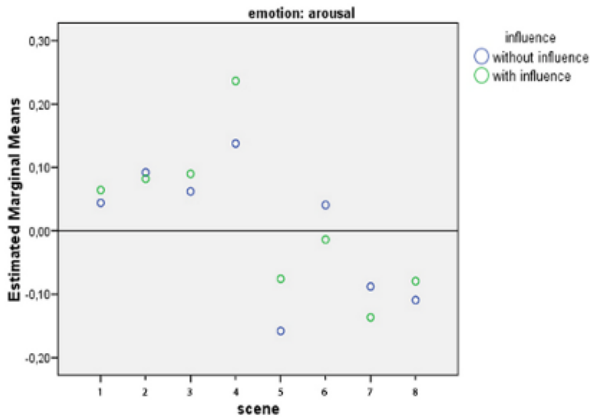

Fig. 4. Estimated means of reported arousal intensity per scene 
were based on intuition. When scenes were selected to represent each pleasurearousal quadrant the aim was to find scenes that were pure in the emotional charge they contained and they were selected intuitively (by two independent researchers) to fall into a certain quadrant of Russell's [34] pleasure-activation model. It is therefore interesting to see that at least to some extent this intuitive mapping corresponded with the emotional rating participants gave to the different scenes, even if not all scenes were equally representative of their quadrant. The data leads one to believe that translation of scenes to pleasure and arousal components is possible to a certain extent. Even if the current research does not show a great effect of bodily influence on emotion, at least this finding is of interest and of possible use in further research.

Another problem was the subtlety of influence, or lack thereof. Technical imperfections in the chair hindered implicit bodily influence and made the user more aware of the fact that they were being influenced than should have been the case. Speed of posture change was too high, and there was more than one source of noise when the various modalities were activated, confusing and even annoying the participants. Although it produced noise and could be clearly felt throughout the chair, heart rate feedback was not noticed as such by most participants, and there was no significant effect on reported heart rate. Whether this was due to the interruption of the experience or if this modality is simply unable to produce the desired effect remains unclear and may be subject for further research.

Finally, a choice in the presentation of the questionnaire led to a possible problem. Instead of asking participants to recall their experienced emotions for all scenes at the end of the experiment, a choice was made to pause between scenes and have them fill out the questionnaire regarding the last viewed scene to prevent difficulties presented by the recollection of emotions. The result was that participants were not only made aware of their bodily state as such, but even of their emotions as the final question was always to describe the emotions they experienced. Even though hardly any of the participants claimed to know the goal of the experiment at the debriefing, some could guess and it is possible that the implicitness of the emotional influence was endangered simply by making users aware of their emotions and their role in the experiment.

Regarding the analysis of the data, a problem was encountered when deriving the pleasure and arousal intensities scored per scene. The questionnaire contained items that scored purely along one single axis, but also items that represented diagonals in the pleasure-arousal domain. In this way, participants were forced to score a scene along a diagonal which may not apply at all to a scene. For example, when faced with a choice between frustration or contentedness for a happy or sad scene, does pleasure or arousal influence the direction in which users will score the scene? As the diagonal enters two quadrants both of which are not applicable to the selected scene, to what extent can the score indicated along this diagonal be regarded as a reliable result? The solution in this case was to consider all scores as vectors in the pleasure-arousal domain and average them. As both axes and both diagonals are represented, the average vector should still occupy the 
correct quadrant. Still, the effect of using these diagonals is not quite clear and should perhaps be avoided in future research in order to get a clearer image of how participants experience a movie scene.

This experiment encountered several problems that may have influenced the outcome. These problems consisted mainly of technical issues that hindered the immersion of the participants' experience. On top of that, many will agree that it is difficult to gather unambiguous empirical data when working with people and subjectively experienced emotions.

In conclusion we can say that, in spite of the problems encountered, the fact that movie scenes can be considered in terms of pleasure and arousal is in itself interesting. The main hypothesis of this research is not supported by these results though, but by no means does this indicate that it is not possible to enhance an immersive experience through induction of bodily states specific to certain emotional states.

\section{Future Work}

More research can provide us with more insight into the possibilities of applying William James' theory of emotion in the entertainment industry.

Some changes should be made to this experiment setup to ensure more reliable and possibly useful data. Such changes would include (1) the use of many more participants, (2) fewer scenes, perhaps only one per quadrant or two per axis, and fewer scenes per subject, (3) blind rating of the emotional charge of selected scenes by a group of independent reviewers prior to experimentation, (4) fewer and clearer questionnaire items in terms of their meaning in pleasure-arousal terms, (5) more subtle influence in terms of noise and speed of position changes.

Regarding the various modalities, some considerations as to the exact implementation should be made as well. In this experiment the chair was fitted with air ventilation to lower the temperature because, among other reasons, it was the quickest and cheapest solution to create. Some people did notice the air flow however and of course the noise from a slow moving fan is an issue. Perhaps a cooling system integrated in the back and seat of the chair would be more functional. Peltier elements may be used for heating as well as cooling if budget allows. In terms of position adjustment, a speed-adjustable, programmable motor with Hall-sensor for position feedback would be the wise choice. As for heart rate feedback, perhaps smaller, silent vibrating elements can be used in the back and seat, although one single vibrating motor on the back was enough to feel a vibration through the entire chair. A silent version of this solution would work well. Finally, other modalities as described earlier can be added as well, although for the purposes of clear and unambiguous results we would advise against an abundance of modalities. Perhaps the various modalities should even be tested separately, in separate experiments, to determine their effectiveness in this field. 


\section{Acknowledgements}

The authors wish to thank Rob Overkamp and Daniel Attevelt for extensive technical support, Arjen van der Meulen for providing and aiding in the selection of video material and Sofie Brandsteder for her great help with statistical analysis.

\section{References}

1. W. James, What is an emotion?, Mind, Vol. 9 (1884), pp. 188-205.

2. P. Philippot, G. Chapelle, S. Blairy, Respiratory feedback in the generation of emotion, Cognition and Emotion, Vol. 16 (2002) No. 5, pp. 605-627

3. S.E. Duclos, J.D. Laird, E. Schneider, M. Sexter, L. Stern, O. Van Lighten, Emotionspecific effects of facial expressions and postures on emotional experience, Journal of Personality and Social Psychology, Vol. 57 (1989) No. 1, pp. 100-108

4. D.N. McIntosh, R.B. Zajonc, P.S. Vig, S.W. Emerick, Facial Movement, Breathing, Temperature and Affect: Implications of the Vascular Theory of Emotional Efference, Cognition and Emotion, Vol. 11 (1997) No. 2, pp. 171-195

5. J.H. Riskind, C.C. Gotay, Physical Posture: Could It Have Regulatory or Feedback Effects on Motivation and Emotion?, Motivation and Emotion, Vol. 6 (1982) No. 3, pp. 273298

6. F.A. Boiten, N.H. Frijda, C.J.E. Wientjes, Emotions and respiratory patterns: review and critical analysis, International Journal of Psychophysiology, Vol. 17 (1994), pp. 103-128

7. B. Mittelmann, H.G. Wollf, Affective States and Skin Temperature: Experimental Study of Subjects With "Cold Hands" and Raynaud's Syndrome, Psychosomatic Medicine, Vol. 1 (1939) No. 2, pp. 271-292

8. E. Briese, Emotional Hyperthermia and Performance in Humans, Physiology \& Behavior, Vol. 58 (1995) No. 3, pp. 615-618

9. R.A. McFarland, Relationship of Skin Temperature Changes to the Emotions Accompanying Music, Biofeedback and Self-Regulation, Vol. 10 (1985) No. 3, pp. 255-267

10. C.A. Anderson, Temperature and Aggression: Ubiquitous Effects of Heat on Occurrence of Human Violence, Psychological Bulletin, Vol. 106 (1989) No. 1, pp. 74-96

11. C.A. Anderson, K.B. Anderson, W.E. Deuser, Examining an Affective Aggression Framework: Weapon and Temperature Effects on Aggressive Thoughts, Affect and Attitudes, Personality and Social Psychology Bulletin, Vol. 22 (1996) No. 4, pp. 366-376 
12. H.Z. Tan, A. Pentland, Tactual Displays for Wearable Computing, Personal Technologies, Vol. 1 (1997), pp. 225-230

13. S. Brave, A. Dahley, inTouch: A Medium for Haptic Interpersonal Communication, CHI 97, (1997) 22-27 March, pp. 363-364

14. D. Mathew, vSmileys: Imaging Emotions through Vibration Patterns, Alternative Access: Feelings and Games 2005, (2005) Spring

15. A.F. Rovers, H.A. Van Essen, HIM: A Framework for Haptic Instant Messaging, CHI 2004, (2004) April 24-29, pp. 1313-1316

16. S. Yohanan, M. Chan, J. Hopkins, H. Sun, K. MacLean, Hapticat: Exploration of Affective Touch, ICMI'05, (2005) October 4-6, pp. 222-22

17. M.D.Decaria, S. Proctor, T.E. Malloy, The effect of false heart rate feedback on selfreports of anxiety and on actual heart rate, Behavior Research \& Therapy, Vol. 12 (1974), pp. 251-253

18. K.T. Larkin, S.B. Manuck, A.L. Kasprowicz, The Effect of Feedback-Assisted Reduction in Heart Rate Reactivity on Videogame Performance, Biofeedback and self-regulation, Vol. 15 (1990) No. 4, pp. 285-303

19. E.H. Liebhart, Effects of False Heart Rate Feedback and Task Instructions on Information Search, Attributions, and Stimulus Ratings, Psychological Research, Vol. 39 (1977), pp. 185-202

20. B. Parkinson, L. Colgan, False Autonomic Feedback: Effects of Attention to Feedback on Ratings of Pleasant and Unpleasant Target Stimuli, Motivation and Emotion, Vol. 12 (1988) No.1, pp. 87-98

21. C. Collett, E. Vernet-Maury, G. Delhomme, A. Dittmar, Autonomic nervous system response patterns specificity to basic emotions, Journal of the Autonomic Nervous System, Vol. 62 (1997), pp. 45-57

22. H.D. Critchley, R. Elliott, C.J. Mathias, R.J. Dolan, Neural Activity Relating to Generation and Representation of Galvanic Skin Conductance Responses: A Functional Magnetic Resonance Imaging Study, The Journal of Neuroscience, Vol. 20 (2000) No. 8, pp. 3033-3040

23. H.D. Critchley, Electrodermal Responses: What Happens in the Brain, Neuroscientist, Vol. 8 (2002) No. 2, pp. 132-142

24. W. Hubert, R. De Jong-Meyer, Psychophysiological Response Patterns to Positive and Negative Film Stimuli, Biological Psychology, Vol. 31 (1990), pp. 73-93

25. S. Khalfa, P. Isabelle, B. Jean-Pierre, R. Manon, Event-related skin conductance responses to musical emotions in humans, Neuroscience Letters, Vol. 328 (2002), pp. 145149 
26. A. Pecchinenda, C.A. Smith, The Affective Significance of Skin Conductance Activity During a Difficult Problem-Solving Task, Cognition and Emotion, Vol. 10 (1996) No. 5, pp. $481-503$

27. A.J. Ferreira, W.D. Winter, The Palmar Sweat Print: A Methodological Study, Psychosomatic Medicine, Vol. 22 (1963) No. 4, pp. 377-384

28. B. Mittelmann, H.G. Wollf, Affective States and Skin Temperature: Experimental Study of Subjects With "Cold Hands" and Raynaud's Syndrome, Psychosomatic Medicine, Vol. 1 (1939) No. 2, pp. 271-292

29.J.A. Allen, J.E. Armstrong, I.C. Roddie, The Regional Distribution of Emotional Sweating in Man. Journal of Physiology, Vol. 235 (1973) No. 3, pp. 749-759

30. G. Epple, R.S. Herz, Ambient Odors Associated to Failure Influence Cognitive Performance in Children, Developmental Psychobiology, Vol. 35 (1999), pp. 103-107

31. R.S. Herz, J. Eliassen, S. Beland, T. Souza, Neuroimaging evidence for the emotional potency of odor-evoked memory, Neuropsychologia, Vol. 42 (2004), pp. 371-378

32. A.N. Rétiveau, E. Chambers IV, G.A. Milliken, Common and Specific Effects of Fine Fragrances on the Mood of Women, Journal of Sensory Studies, Vol. 19 (2004), pp. 373394

33. J. Haviland-Jones, D.J. McGuire, The Scents of Fear and Funny, The Aroma-Chology Review, Vol. 8 (1999) No. 2, pp. 11

34. J.A. Russell, Core Affect and the Psychological Construction of Emotion, Psychological Review, Vol. 110 (2003) No. 1, pp. 145-172 